\title{
Effects of deformation in the three-body structure of ${ }^{11} \mathrm{Li}$
}

\author{
I. Brida, F.M. Nunes and B.A. Brown ${ }^{\text {a }}{ }^{*}$
}

${ }^{a}$ NSCL and Department of Physics and Astronomy Michigan State University, East Lansing MI 48824 USA

${ }^{11} \mathrm{Li}$ is studied within a three-body model ${ }^{9} \mathrm{Li}+n+n$ where the core is allowed to be deformed and/or excite. In particular, we include reorientation couplings and couplings between the two bound states of ${ }^{9} \mathrm{Li}$. Contrary to the other examples studied within this model, we find that core excitation does not affect the structure of ${ }^{11} \mathrm{Li}$ significantly. Reorientation couplings of the deformed ${ }^{9} \mathrm{Li}$ can change the ground state of ${ }^{11} \mathrm{Li}$ from a predominantly two neutron $\left(s_{1 / 2}\right)^{2}$ configuration into a $\left(p_{1 / 2}\right)^{2}$. In addition, we see no evidence for the existence of significant d-wave strength in its ground state, as opposed to the prediction by shell model. A comparison with shell model is presented.

\section{Introduction}

The prima donna of all halo nuclei is ${ }^{11} \mathrm{Li}$ : it offers an exciting experimental challenge but also questions the basic concepts of a large variety of structure theories. Many experiments have been performed over the last decade, since the pioneering total reaction cross section experiment at Berkeley [1. These include Coulomb dissociation experiments 23], mass measurements [4.5], momentum distributions of the ${ }^{9} \mathrm{Li}$ coming from the two neutron removal [6 7 8 and of ${ }^{10} \mathrm{Li}$ coming from the one neutron removal 9 10, proton inelastic scattering [11, as well as beta decay studies [12 1314]. We now know the system is bound by $295 \pm 35 \mathrm{keV}$ against three-body breakup [4] with a momentum distribution around $40 \mathrm{MeV} / \mathrm{c}$ [7] (see Addendum at the end of the paper). The ground state structure contains two valence neutrons in $\left(s_{1 / 2}\right)^{2}$ and $\left(p_{1 / 2}\right)^{2}$ configurations with approximately equal probability [10 12]. The r.m.s. matter radius of the system is $3.55 \pm 0.10 \mathrm{fm}$ [15].

One cannot understand the Borromean nucleus ${ }^{11} \mathrm{Li}$ without a good description of the $n$-core subsystem ${ }^{10} \mathrm{Li}$. Experimental programs are aware of this fact but, as this nucleus is particle unbound, it presents a further technical obstacle. The information on ${ }^{10} \mathrm{Li}$ is summarized in [16]. Therein, it is possible to see the large number of experiments that have been performed to measure ${ }^{10} \mathrm{Li}$ 's spectrum, but also the contradictory energy, parity and spin assignments made. Overall one can conclude the following: (i) the ground state of ${ }^{10} \mathrm{Li}$ contains a valence neutron outside the $p_{3 / 2}$ subshell in a $s_{1 / 2}$ state, at approximately $50 \mathrm{keV}$ or below; (ii) there is a state around $0.4 \mathrm{MeV}$ that can be understood as a neutron single particle excitation into the $p_{1 / 2}$ orbital; (iii) there is no clear evidence for a d-state below $3 \mathrm{MeV}$.

\footnotetext{
*This work is supported by by the National Science Foundation through grants PHY-0456656 and PHY-
} 0244453, and the NSCL at Michigan State University. 
In the early days, three-body models of two neutrons and an inert ${ }^{9} \mathrm{Li}$ core were developed to describe properties of ${ }^{11} \mathrm{Li} 17181920$. At that time nothing was known about ${ }^{10} \mathrm{Li}$, and theorists could play the game of adjusting freely the $n-{ }^{9} \mathrm{Li}$ interaction in order to produce a sensible ${ }^{11} \mathrm{Li}$ ground state [181921. Models produced states where the two neutrons were in $\left(2 s_{1 / 2}\right)^{2}$ and in $\left(1 p_{1 / 2}\right)^{2}$ states coupled to the ground state of the core ${ }^{9} \mathrm{Li}$. The relative strength of these two components varied significantly depending on the $n$-core effective interaction. In 21] a three-body force was introduced in addition to the two-body $n-n$ and $n$-core interactions. In [1720] a density dependent $n-n$ delta force was used and emphasis was given to the importance of pairing. Three-body inert core models have been expanded to generate three-body continuum states [22] and the complexity of these three-body scattering states was analyzed within the context of proton inelastic scattering.

Microscopic calculations in the early nineties were unable to reproduce a realistic binding energy for ${ }^{11} \mathrm{Li}$ without artificially renormalizing the interactions, namely forcing the $p_{1 / 2}$ orbital to be closer to the continuum threshold (e.g. 2324]). As pairing effects had been identified to be crucial [17, by introducing a phenomenological force in the pairing channel, a self consistent description of the Li isotopic chain became possible within the relativistic Hartree-Bogolyubov framework (e.g. [25]). Effective interactions valid near the driplines have meanwhile been developed in shell model [26]. Nevertheless, configuration mixing, required to produce a realistic ground state for ${ }^{11} \mathrm{Li}$, is still introduced by hand. It is only recently that ab-initio methods have reached mass $A=11$ [27 28]. Although the general spectra look promising, there is a clear limitation on the accuracy of the energy (and consequently of the two neutron separation energy) and much more effort is necessary to obtain reliable spectroscopic information.

There are still some open questions regarding ${ }^{11} \mathrm{Li}$, even when considering the ground state only. Should the quadrupole deformation of ${ }^{9} \mathrm{Li}$ play a role in the structure of ${ }^{11} \mathrm{Li}$ ? Assuming the low lying states of ${ }^{10} \mathrm{Li}$ are mostly neutron single particle excitations, then we should find a d-wave around $2 \mathrm{MeV}$ excitation energy, as in the case of ${ }^{11} \mathrm{Be}$. Even if experiment has been inconclusive in this respect, if such a state were to exist, what would be the implications for the ground state of ${ }^{11} \mathrm{Li}$ ? Would one expect to also have a strong $\left(d_{5 / 2}\right)^{2}$ configuration in the ground state of ${ }^{11} \mathrm{Li}$ as in the ground state of ${ }^{12} \mathrm{Be}$ ?

On the issue of core deformation and/or excitation, ${ }^{11} \mathrm{Li}$ is considered within the Nilsson model [29]. The core ${ }^{9} \mathrm{Li}$ has an uncoupled proton that can be either in a $K=3 / 2^{-}$or $K=1 / 2^{-}$orbital. In that simple picture, the extra two neutrons in ${ }^{11} \mathrm{Li}$, occupy a different K-orbit and would not be strongly affected by the orientation of the deformed axis. In addition, a shell model calculation using two independent frequencies (one for the core, the other for the halo neutrons) 30] shows that core polarization due to the halo neutrons is very small.

The three-body model with core excitation 313233 allows us to address these issues keeping all the three-body dynamics. This model was applied to the Be isotopes ${ }^{11} \mathrm{Be}$ and ${ }^{12} \mathrm{Be}$. The coupled channel description of the states in ${ }^{11} \mathrm{Be}$ was produced assuming a ${ }^{10} \mathrm{Be}+\mathrm{n}$ structure where the $2^{+}$excited state of ${ }^{10} \mathrm{Be}$ was explicitly included in the model space. A rotational model was used for the core but the strength of the coupling between the two ${ }^{10} \mathrm{Be}$ states was taken from the measured $B(E 2)$ value. An effective $n+$ core interaction for ${ }^{11} \mathrm{Be}$ could be made to reproduce the parity inversion of the $1 / 2^{+}$ 


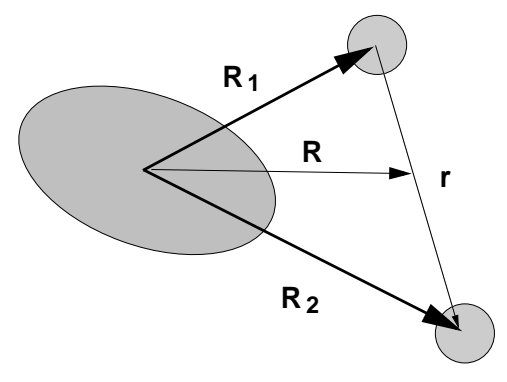

Figure 1. Representation of the relevant coordinates in the model.

and $1 / 2^{-}$bound states, the spectroscopy of these states, as well as the $\mathrm{d}$-wave resonance. Based on such a realistic description of the ${ }^{10} \mathrm{Be}+\mathrm{n}$ subsystem [31] three-body calculations were performed using hyper-spherical coordinates and a hyper-spherical harmonic basis [32. In this formulation, the Faddeev equations reduce to a set of hyper-radial coupled channel equations. Antisymmetrization was taken into account in an approximate way by projecting out the lowest ${ }^{10} \mathrm{Be}+\mathrm{n}$ states with large overlap with the occupied $1 s_{1 / 2}$ and $1 p_{3 / 2}$. Our model was able to predict most features of the ${ }^{12} \mathrm{Be}$ system [33. In particular, the ground state was predicted to contain roughly one third of core excitation with neutrons in the $\left(d_{5 / 2}\right)^{2}$.

If only the neutrons play a role in the low lying spectra of ${ }^{10} \mathrm{Li}$ and ${ }^{11} \mathrm{Li}$, one expects similarities between the spectra of these nuclei and those of ${ }^{11} \mathrm{Be}$ and ${ }^{12} \mathrm{Be}$. It is then reasonable to think of ${ }^{10} \mathrm{Li}$ as ${ }^{9} \mathrm{Li}+\mathrm{n}$ and of ${ }^{11} \mathrm{Li}$ as ${ }^{9} \mathrm{Li}+\mathrm{n}+\mathrm{n}$. The main difference between the three-body model of ${ }^{9} \mathrm{Li}+\mathrm{n}+\mathrm{n}$ and that of ${ }^{10} \mathrm{Be}+\mathrm{n}+\mathrm{n}$ is the spin of the ground state of the core. Exactly for this reason, computational needs for ${ }^{11} \mathrm{Li}$ are much larger than for ${ }^{12} \mathrm{Be}$. Also, the first excited state of ${ }^{10} \mathrm{Be}\left(2^{+}\right)$couples the valence neutrons to new orbitals whereas the ground state of ${ }^{9} \mathrm{Li}\left(3 / 2^{-}\right)$enables a larger variety of orbitals than its excited state $1 / 2^{-}$. Note that, in ${ }^{9} \mathrm{Li}$, there are reorientation couplings just for the ground state, which are not present in ${ }^{10} \mathrm{Be}$. Are these differences important when modelling ${ }^{11} \mathrm{Li}$ ? Now that computational power is very much increased, it is timely to revisit the problem under the constraints provided by all the new ${ }^{10} \mathrm{Li}$ data.

\section{Ingredients for the calculations}

The Hamiltonian in the three-body model with core excitation can be written as:

$H_{3 B}=T_{R}+T_{r}+V_{n n}(\vec{r})+V_{n c}\left(\overrightarrow{R_{1}}, \xi\right)+V_{n c}\left(\overrightarrow{R_{2}}, \xi\right)+h_{c}(\xi)$

where the coordinates are represented in Fig. 1 1 and $\xi$ is an internal degree of freedom of the core. These coordinates are transformed into the hyper-spherical coordinates and through an appropriate expansion of the wavefunction, the three-body Schrödinger equation is reduced to a set of coupled channel equations dependent on hyper-radius $\rho$ and hypermomentum $K$. The truncation of the basis is done through the quantum number $K$. Other details of the calculations can be found in [32 35]. 


\begin{tabular}{|l|r|}
\hline$J^{\pi}$ & ${ }^{11} \mathrm{Be}$ \\
\hline $1 / 2^{+}$ & 0.0 \\
$1 / 2^{-}$ & 0.32 \\
$5 / 2^{+}(?)$ & 1.78 \\
$3 / 2^{-}(?)$ & 2.69 \\
\hline
\end{tabular}

Table 1

Low lying spectrum of ${ }^{11} \mathrm{Be}$ [34]. Excitation energies in $\mathrm{MeV}$.

Here we discuss the physical inputs: the interactions and the model for the core. The interaction between the two neutrons $V_{n n}$ is usually taken from a parameterization of the low energy nucleon-nucleon phase shifts, which are well understood. We use GPT interaction 36, neglecting the LL term. Most ambiguities reside in pinning down the effective interaction between the core ${ }^{9} \mathrm{Li}$ and each neutron. We will use the notation $n l_{j} \otimes I$ to denote a specific $n$ - core configuration. For example $1 p_{1 / 2} \otimes 3 / 2^{-}$corresponds to a valence $1 p_{1 / 2}$ neutron coupled to the $3 / 2^{-9} \mathrm{Li}$ ground state. A Woods-Saxon form plus spin-orbit is fitted to reproduce three main structures in ${ }^{10} \mathrm{Li}$, namely the virtual state at $+50 \mathrm{keV}$ with configuration $2 s_{1 / 2} \otimes 3 / 2^{-}$, the resonance at $+400 \mathrm{keV}$ with configuration $1 p_{1 / 2} \otimes 3 / 2^{-}$and a resonance at $+3.4 \mathrm{MeV}$ with configuration $1 d_{5 / 2} \otimes 3 / 2^{-}$. Due to the lack of experimental data, the position of d-resonance in ${ }^{10} \mathrm{Li}$ is not known. By comparison with ${ }^{11} \mathrm{Be}$, with the same number of neutrons, one would expect a resonance close to 2 $\mathrm{MeV}$ excitation (see Table 11). We place it at $+3.4 \mathrm{MeV}$ : if the d-wave resonance were at much higher energy, the binding energy of ${ }^{11} \mathrm{Li}$ would not be reproduced; if it were at much lower energy it would become more bound than a $\mathrm{p}$-wave resonance, for large deformation. Also we consider that the $1 p_{3 / 2}$ orbital is at $-4.1 \mathrm{MeV}$, to match the neutron separation energy of ${ }^{9} \mathrm{Li}$.

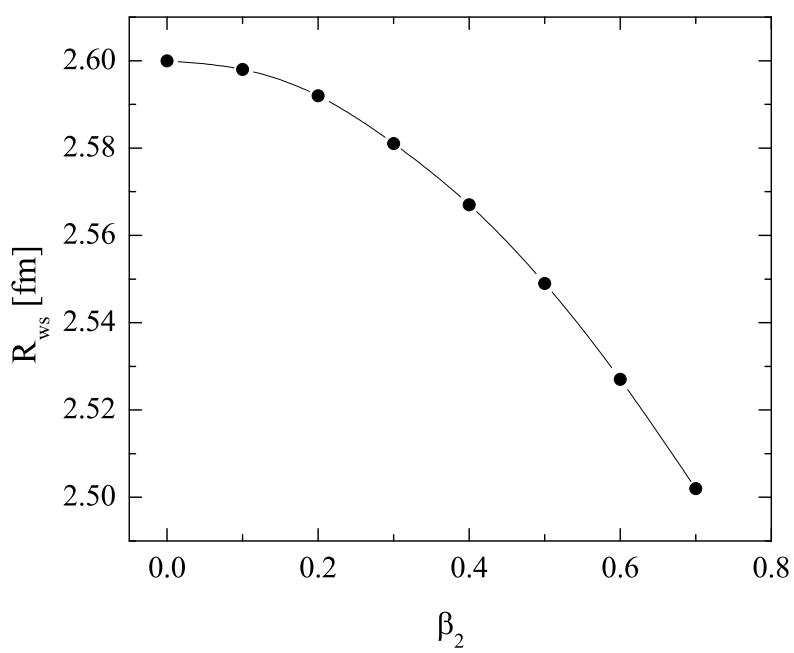

Figure 2. Radii of the fitted $\mathrm{n}-{ }^{9} \mathrm{Li}$ interaction as a function of deformation. 


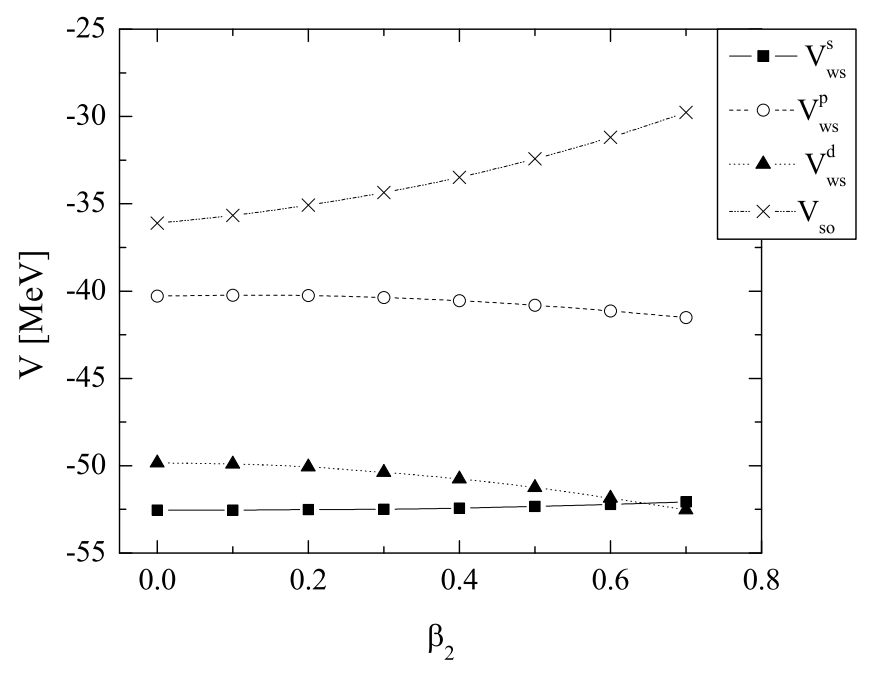

Figure 3. Depths of the fitted $\mathrm{n}^{9} \mathrm{Li}$ interaction as a function of deformation.

When there is deformation and core excitation, $l, j$ are no longer good quantum numbers, and the nuclear state $J^{\pi}$ contains a superposition of different $n l_{j} \otimes I$ configurations. Nevertheless, and for simplicity, we will refer to any multi-component state $J^{\pi}$, by the component into which the state collapses in the limit of no couplings.

The form for the $n$-core interaction is:

$$
V_{n c}\left(\vec{R}_{i}, \xi\right)=\frac{V_{w s}^{l}}{1+e^{\left(\frac{R_{i}-R(\theta, \phi)}{a_{w s}}\right)}}-\left(\frac{\hbar}{m_{\pi} c}\right)^{2}(2 \vec{l} \cdot \vec{s}) \frac{V_{s o}}{4 R_{i}} \frac{d}{d R_{i}}\left[1+e^{\left(\frac{R_{i}-R_{s o}}{a_{s o}}\right)}\right]^{-1}, \quad i=1,2
$$

Quadrupole deformation $\beta_{2}$ serves as the collective degree of freedom for the core introduced in Eq1. The central Woods-Saxon part depends on the deformation through the radius $R(\theta, \phi)=R_{w s}\left(1+\beta_{2} Y_{20}(\theta, \phi)\right)$, where $\theta$ and $\phi$ are spherical angles in a rest frame of the core. The spin-orbit term is left undeformed. In addition, the core is treated within a rotational model, parameterized in terms of $\beta_{2}$. This is an oversimplification of the structure of ${ }^{9} \mathrm{Li}$, and thus we will not impose that the strength of the couplings be determined by the $B(E 2)$, which in any case is not known. Volume conservation, however, is imposed on the central $n$-core interaction [31], so when $\beta_{2} \neq 0$, the radius parameter $R_{w s}$ is adjusted accordingly. We take a standard value $R_{w s}=2.60 \mathrm{fm}$ when $\beta_{2}=0$ and its dependence for $\beta_{2} \neq 0$ is shown in Fig. 2. Radius $R_{\text {so }}$ was made equal to $R_{w s}$ at any deformation. The corresponding diffusenesses are fixed to the standard value $a_{w s}=a_{s o}=0.65 \mathrm{fm}$.

The depth of the central part of the interaction between the core and the neutron depends on their relative angular momentum $l$. We consider $V_{w s}^{s}$ and $V_{w s}^{p}$ for $l=0$ and 1 , respectively and the same $V_{w s}^{d}$ for all partial waves with $l \geq 2$. The depth $V_{s o}$ of the spin-orbit term is $l$-independent. As mentioned before, the depths of $n$-core potentials were fitted in order to reproduce the low lying continuum structure of ${ }^{10} \mathrm{Li}$ as well as the position of bound $1 p_{3 / 2}$ neutron orbital. 


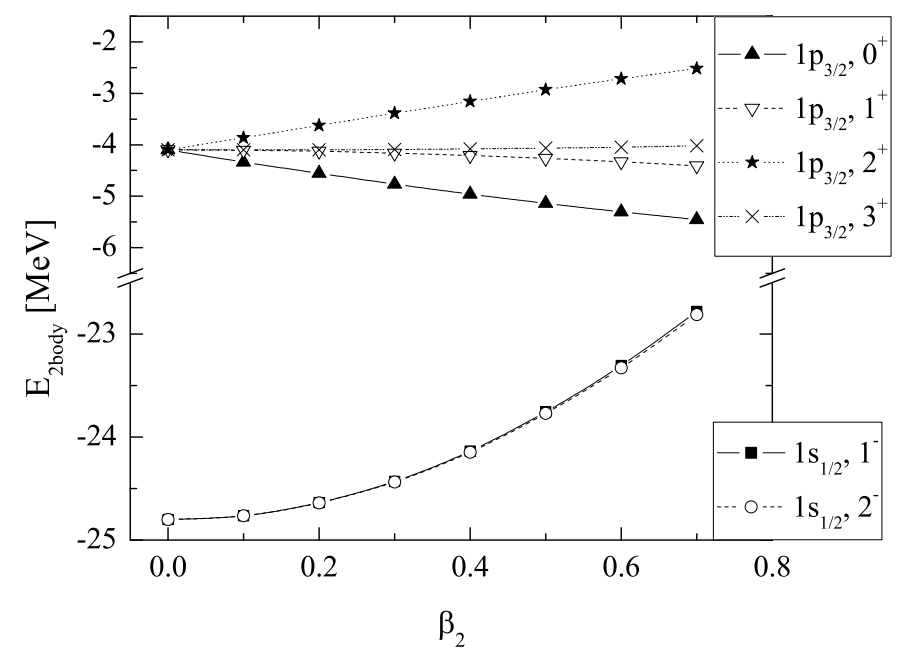

Figure 4. Deeply bound states in ${ }^{10} \mathrm{Li}$ as a function of deformation. The legend refers to $n l_{j}$ valence neutron orbitals (coupled to the $3 / 2^{-}$ground state of ${ }^{9} \mathrm{Li}$ ) and total spin of ${ }^{10} \mathrm{Li}$.

In principle, due to the low excitation energy in ${ }^{9} \mathrm{Li}(2.69 \mathrm{MeV})$, we should include the $1 / 2^{-}$state in our core model. Such calculations were performed but results were not

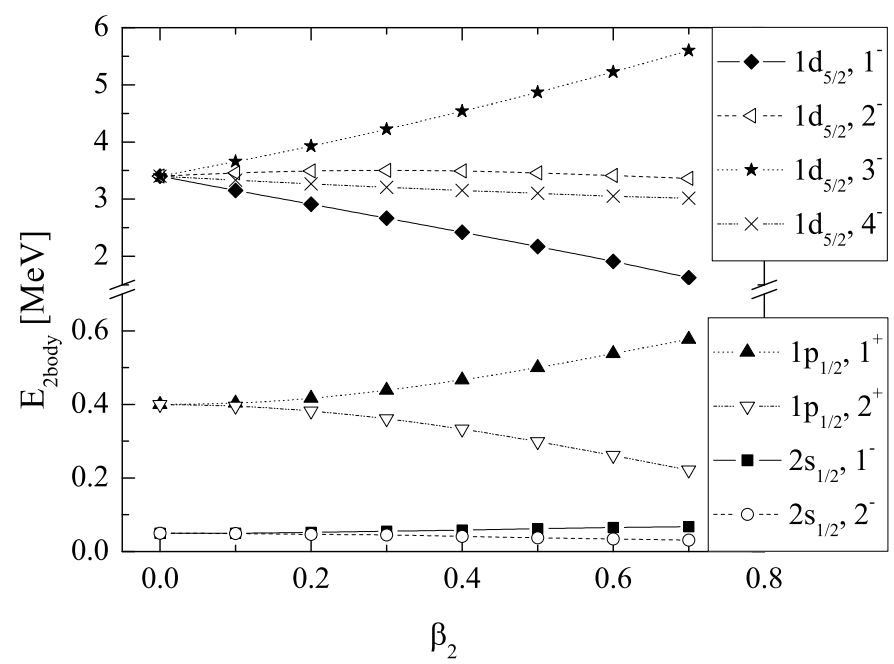

Figure 5. Continuum states in ${ }^{10} \mathrm{Li}$, as a function of deformation. The legend refers to $n l_{j}$ valence neutron orbitals (coupled to the $3 / 2^{-}$ground state of ${ }^{9} \mathrm{Li}$ ) and total spin of ${ }^{10} \mathrm{Li}$. Both s-wave states refer to virtual states while the other states correspond to real resonances. 


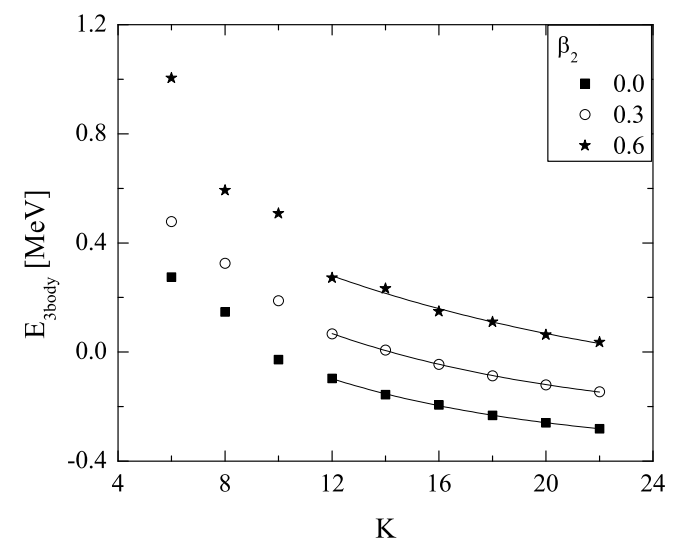

Figure 6. Convergence behaviour for ${ }^{11} \mathrm{Li}$ as a function of the hyper-momentum $\mathrm{K}$. The lines are an exponential fit.

sensitive to the inclusion of this additional state. As mentioned in Section 1 and due to its low spin value, there are no new orbitals brought into the configuration space when including this state. For this reason in ${ }^{11} \mathrm{Li}$ reorientation is significant but core excitation is not. Note that the model space when both core states $\left(3 / 2^{-} ; 1 / 2^{-}\right)$are included increases considerably. We will present results for the case where only the ground state $3 / 2^{-}$of ${ }^{9} \mathrm{Li}$ is taken into account.

In addition, the deformation parameters of ${ }^{9} \mathrm{Li}$ can also take negative values (if the core is oblate). We found that the quadrupole force for oblate shapes, produces more repulsion when compared to the prolate case, and therefore less binding energy. Based on the known positive quadrupole moment of ${ }^{7} \mathrm{Li}$, we expect this to also be positive for ${ }^{9} \mathrm{Li}$. Therefore, we consider always positive deformation for all results discussed in this work.

In case of an undeformed ${ }^{9} \mathrm{Li}$, all $J^{\pi}$ states in ${ }^{10} \mathrm{Li}$ originating from a given $n l_{j}$ neutron coupled to the core are degenerate. The degeneracy is, however, removed as soon as the spherical symmetry is broken by non-zero deformation. In the deformed cases, fitting potentials means to adjust their depths such that the centroids of $1 p_{3 / 2}, 2 s_{1 / 2}, 1 p_{1 / 2}$ and $1 d_{5 / 2}$ orbitals are kept at $-4.1 \mathrm{MeV},+50 \mathrm{keV},+400 \mathrm{keV}$ and $+3.4 \mathrm{MeV}$, respectively. Again here, the $1 d_{5 / 2}$ centroid at $3.4 \mathrm{MeV}$ ensures that even when $\beta_{2}=0.7$ the splitting does not produce inversion of the p-state and the d-state. The variation of potential depths with deformation is shown in Fig. 3. As in ${ }^{11} \mathrm{Be}$, the spin orbit strength needed is large [31. The corresponding two-body bound states and the lowest resonances in ${ }^{10} \mathrm{Li}$ are shown in Fig. 4 and Fig. 5, respectively.

Finally, in order to approximately satisfy the exclusion principle, the bound $1 s_{1 / 2}$ and $1 p_{3 / 2}$ neutron orbitals are projected out of the model space before diagonalization [32].

\section{Results as a function of deformation}

Calculations were performed using EFADD [37. Our model space consists of 18 Laguerre polynomials for the hyper-radial part, reaching out to $\rho_{\max }=20 \mathrm{fm}$, and $30 \mathrm{Jacobi}$ 


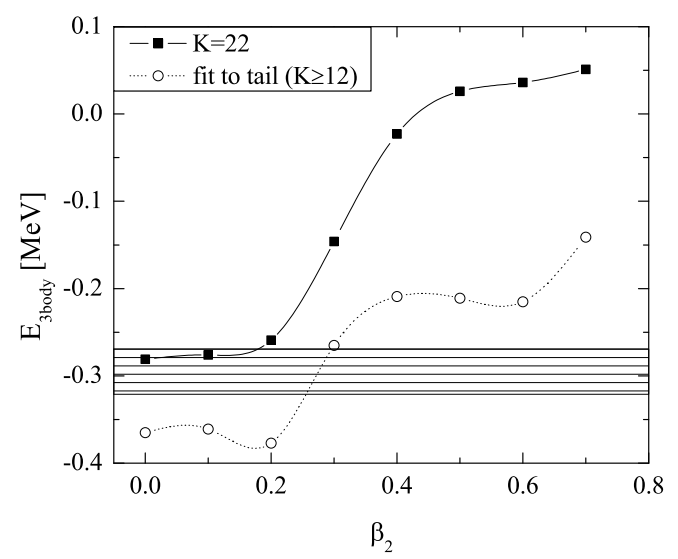

Figure 7. Three-body energy of the ground state of ${ }^{11} \mathrm{Li}$ as a function of deformation. Shaded region corresponds to data [4]

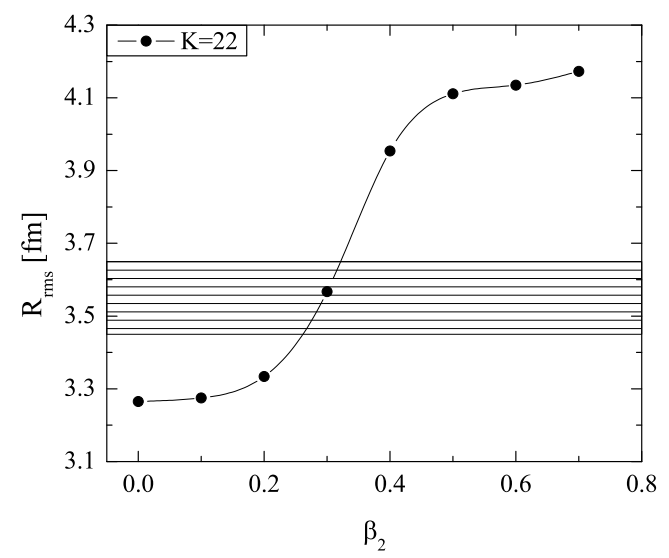

Figure 8. Radius of ${ }^{11} \mathrm{Li}$ as a function of deformation. Shaded region corresponds to the value consistent with reaction data [15]

polynomials for the hyper-angular part. Calculations were performed up to $K_{\max }=22$.

In Fig. 6] we show the convergence of the three-body binding energy of ${ }^{11} \mathrm{Li}$, for $\beta_{2}=$ 0.0, 0.3 and 0.6, as a function of hyper-momentum. On one hand, the convergence exhibits the well known exponential dependence when $K_{\max } \gtrsim 12$ for all deformations studied. On the other hand, in all cases, the convergence rate is very slow, much slower than in the ${ }^{12} \mathrm{Be}$ case (see Fig. 6 of [32]). Moreover, the convergence rate decreases with increasing deformation. It is thus necessary to use the extrapolated energy value for an accurate binding energy. We show results up to $\beta_{2}=0.7$ (this value is already unrealistically large).

Results for the three-body energy of ${ }^{11} \mathrm{Li}$ are presented in Fig. 7 . We plot both the values for $K_{\max }=22$ and those obtained through the extrapolation in K. Contrary to 


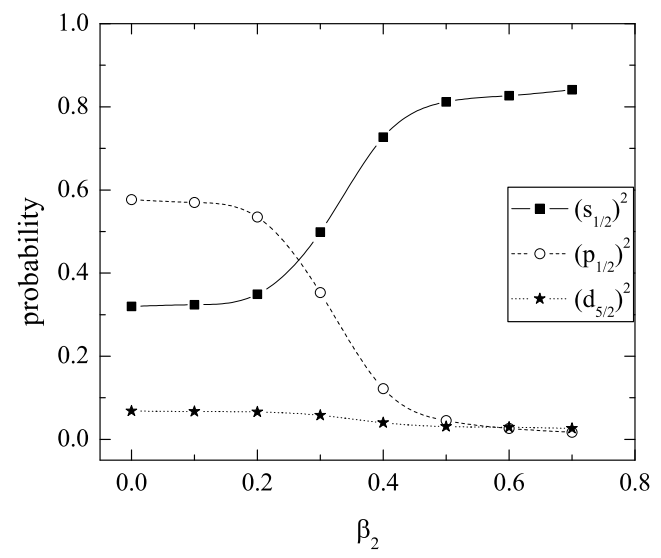

Figure 9. Structure of the ground state of ${ }^{11} \mathrm{Li}$ as a function of deformation.

the case of ${ }^{12}$ Be where the energy gain was large, here a very small additional binding is obtained from the quadrupole coupling, and instead, as the deformation becomes large, the system becomes less bound. The r.m.s. radii of the corresponding wavefunctions are shown in Fig. 8. Here we have used $2.32 \mathrm{fm}$ for the r.m.s. radius $\left(R_{r m s}\right)$ of the ${ }^{9} \mathrm{Li}$ core 38. These radii were obtained with $K_{\max }=22$, but the $R_{r m s}$ variation between $K_{\max }=20$ and $K_{\max }=22$ is less than $1 \%$. Our prediction for the charged radius obtained with $\beta_{2}=0.3$ is $R_{c h}=2.49 \mathrm{fm}$, in agreement with the very recent measurement [39]. The experimental two-neutron separation energy and the r.m.s. matter radius impose a constraint on $\beta_{2} \lesssim 0.3$. Note that the extrapolation was only done for extracting converged energies, since it is the only observable that has a well established exponential dependence on $K_{\max }$.

We now look at the structure of the state. In Fig. 9] we show the probabilities associated with the three main components of the system, namely $\left(s_{1 / 2}\right)^{2},\left(p_{1 / 2}\right)^{2}$ and $\left(d_{5 / 2}\right)^{2}$. If $\beta_{2}$ is small, the ground state of ${ }^{11} \mathrm{Li}$ is almost $60 \%\left(p_{1 / 2}\right)^{2}$; for large $\beta_{2}$, it becomes more than $80 \%\left(s_{1 / 2}\right)^{2}$. The region around $\beta_{2}=0.3$ corresponds exactly to the transition between these two configurations and both are populated with equal probability. Regardless of the deformation, the $\left(d_{5 / 2}\right)^{2}$ configuration is always small $(<7 \%)$. This result is in contrast with the three-body calculations for ${ }^{12}$ Be in [33] where the $\left(d_{5 / 2}\right)^{2}$ configuration was $\approx 30 \%$.

Finally, one has to realize that there is no unique parameterization for the effective $n$-core interaction. We have convinced ourselves that the features shown here for the structure of this three-body system, do not result from a specific parameterization. Other parameterizations, using other radii or spin-orbit parameters, produce exactly the same features. These emerge from the constraints of continuum states in ${ }^{10} \mathrm{Li}$. Variations on these constraints are considered at the end of the next section. 


\begin{tabular}{|c|r|r|r|}
\hline$J^{\pi}$ & 3-body & shell model & experiment \\
\hline $0^{+}$ & 0.0 & 0.0 & 0.0 \\
$2^{+}$ & 2.1 & 1.82 & 2.11 \\
$0^{+}$ & 2.2 & 0.37 & 2.24 \\
$1^{-}$ & 2.6 & 2.62 & 2.73 \\
\hline
\end{tabular}

Table 2

Spectrum for ${ }^{12} \mathrm{Be}$ : comparing three-body results with shell model and experiment 33 . Excitation energies in $\mathrm{MeV}$.

\section{Comparison with shell model}

One may expect ${ }^{11} \mathrm{Li}$ to have a similar structure to ${ }^{12} \mathrm{Be}$. Three-body calculations for ${ }^{12} \mathrm{Be}$ have been compared with data and shell model in the p-sd shell using the WBP interaction. The results for the first low lying states of ${ }^{12} \mathrm{Be}$ from [33] are presented in Table 2. As can be seen, both models are able to reproduce the first $0^{+}, 2^{+}$and $1^{-}$states very well. Not shown, the two models also agree on the structure of the ground state of ${ }^{12} \mathrm{Be}$. A three-body versus shell-model comparison of spectra for ${ }^{11} \mathrm{Li}$ is not easy because ${ }^{11} \mathrm{Li}$ excited states are two-particle unbound.

For the shell-model structure of ${ }^{11} \mathrm{Li}$ we use the WBP Hamiltonian of [40]. The WBP Hamiltonian is applicable to pure $N \hbar \omega$ model-space truncations and is determined from a least squares fit of potential-model interaction strengths to experimental states which are relatively pure $0 \hbar \omega, 1 \hbar \omega$ and $2 \hbar \omega$ in structure. Application of this model to ${ }^{12} \mathrm{Be}$ and ${ }^{11} \mathrm{Li}$ gives $0 \hbar \omega$ and $2 \hbar \omega$ states which are degenerate within $100 \mathrm{keV}$. This implies wavefunctions for both ${ }^{12} \mathrm{Be}$ and ${ }^{11} \mathrm{Li}$ which are $50 \%(s)^{4}(p)^{A-4}(0 \hbar \omega)$ plus $50 \%(s)^{4}(p)^{A-2}(s d)^{2}(2 \hbar \omega)$ and other small amplitudes.

In shell model, the $0 \hbar \omega^{11} \mathrm{Li}$ wavefunction must have 2 neutrons in the $p_{1 / 2}$ orbit while for the $0 \hbar \omega{ }^{9} \mathrm{Li}$ it predicts 0.79 neutrons in the $p_{1 / 2}$ orbit. This means that ${ }^{9} \mathrm{Li}$ is not a $p_{3 / 2}$ closed core, and consequently, the $0 \hbar \omega{ }^{11} \mathrm{Li}$ wavefunction does not look like $0 \hbar \omega{ }^{9} \mathrm{Li}$ plus two $p_{1 / 2}$ neutrons. The excitation energy of the $1 / 2^{-9} \mathrm{Li}$ state predicted by shell model is lower than the experimental value $(1.395 \mathrm{MeV}$ to be compared with the experimental value of $2.69 \mathrm{MeV}$ ). The $2 \hbar \omega{ }^{11} \mathrm{Li}$ wavefunction has the structure of two-neutrons in the sd-shell outside the ${ }^{9} \mathrm{Li}$ core, with occupancies of $50 \%, 29 \%$ and $18 \%$ for $s_{1 / 2}, d_{5 / 2}$ and $d_{3 / 2}$ respectively.

The $0 \hbar \omega$ (negative parity) and $1 \hbar \omega$ (positive parity) spectrum for ${ }^{10} \mathrm{Li}$ with WBP, up to $4 \mathrm{MeV}$, is presented in Table 3, of which the first doublet $2^{-}, 1^{-}$corresponds to $s_{1 / 2}$ and the second doublet $1^{+}, 2^{+}$to $p_{1 / 2}$ strength. The lowest state coming from the $d_{5 / 2}$-wave quadruplet is at $E_{x}\left(4^{-}\right)=2.05 \mathrm{MeV}$, much lower than the energy we considered in the three-body calculations $(3.4 \mathrm{MeV})$.

For the ground state of ${ }^{11} \mathrm{Li}$, shell model predicts $15 \% \mathrm{~d}$-wave. This $15 \% \mathrm{~d}$-component in ${ }^{11} \mathrm{Li}$ comes mainly from the low lying d-waves present in the ${ }^{10} \mathrm{Li}$ spectrum.

Also relevant for the discussion is the overlap $\left\langle{ }^{11} \operatorname{Li}(\right.$ g.s. $)\left|\left(a^{\dagger}, a^{\dagger}\right)_{L}\right|{ }^{9} \mathrm{Li}$ (g.s) $\rangle$ which is predicted to be very strong and nearly exhausts all the spectroscopic strength. This is a clear indication that the first excited state of ${ }^{9} \mathrm{Li}$ is not important in the description of the ground state of ${ }^{11} \mathrm{Li}$.

It is important to understand the implications of the ${ }^{10} \mathrm{Li}$ structure on ${ }^{11} \mathrm{Li}$. Albeit the 


\begin{tabular}{|l|r|}
\hline$J^{\pi}$ & $E_{x}$ \\
\hline $2^{-}$ & 0.00 \\
$1^{+}$ & 0.23 \\
$1^{-}$ & 1.11 \\
$2^{+}$ & 1.15 \\
$0^{-}$ & 1.73 \\
$0^{+}$ & 1.74 \\
$4^{-}$ & 2.05 \\
$1^{-}$ & 2.56 \\
$1^{+}$ & 3.14 \\
$2^{-}$ & 3.25 \\
$2^{+}$ & 3.29 \\
$1^{-}$ & 3.69 \\
$3^{-}$ & 3.74 \\
\hline
\end{tabular}

Table 3

Shell model spectrum for ${ }^{10} \mathrm{Li}$. Excitation energies in $\mathrm{MeV}$.

large number of experiments, a close study of [16] raises questions about the precision with which states in ${ }^{10} \mathrm{Li}$ are known. We have explored the possibility of different assumptions for the states to which we fit the $n-$ core interaction, namely $s_{1 / 2} \otimes 3 / 2^{-}$at $+50 \mathrm{keV}$; $p_{1 / 2} \otimes 3 / 2^{-}$at $+400 \mathrm{keV}$ and $d_{5 / 2} \otimes 3 / 2^{-}$at $+3.4 \mathrm{MeV}$. Of these, the least uncertain is the ${ }^{10} \mathrm{Li}$ ground state. We have checked that the main features of the present work are not changed by moving the p-wave to $500 \mathrm{keV}$. More important is the uncertainty in the location of the d-resonance. There is no clear experimental evidence for a d-wave at +3.4 $\mathrm{MeV}$, or at any lower energy. If it is a broad resonance, or it is superposed by other states belonging to the doublets, it could be much harder to observe experimentally. The width of the d-wave resonance increases rapidly with energy. If a ${ }^{10} \mathrm{Li} \mathrm{d}$-state were at $1.2 \mathrm{MeV}$ as in ${ }^{11} \mathrm{Be}$, the width would be $150 \mathrm{keV}$. The ${ }^{10} \mathrm{Li} E_{x}=2.05 \mathrm{MeV}$ predicted by shell model has $\Gamma=420 \mathrm{keV}$ and if it were shifted to $E_{x}=3.4 \mathrm{MeV}$ as in the three-body model, the width would become $\Gamma=1.2 \mathrm{MeV}$. Such a broad state would be very hard to measure.

As seen above, shell model predicts a $4^{-} \mathrm{d}$-wave state at around $2 \mathrm{MeV}$. What happens in the three-body model if the d-wave is pushed down? We refitted the $n$-core interaction with $\beta_{2}=0.3$, fixing the centroid of the d-resonance at $2.5 \mathrm{MeV}$. The quadruplet states $\left(1^{-}, 2^{-}, 3^{-}, 4^{-}\right)$move from $(2.67 ; 3.50 ; 4.22 ; 3.21)$ to $(1.79 ; 2.53 ; 3.32 ; 2.36)(\mathrm{MeV})$, respectively. An immediate consequence is the additional binding in the ${ }^{11} \mathrm{Li}$ system $(\approx 150 \mathrm{keV})$. However this extra d-wave attraction is not sufficient to change the structure of ${ }^{11} \mathrm{Li}$ 's ground state, which remains essentially $\left(s_{1 / 2}\right)^{2}$ and $\left(p_{1 / 2}\right)^{2}$, with $\left(d_{5 / 2}\right)^{2}$ only up to $\approx 10 \%$.

\section{Conclusions}

We perform three-body calculations for ${ }^{11} \mathrm{Li}$ including deformation and excitation of ${ }^{9} \mathrm{Li}$. We find that reorientation effects of the core can account for the known configuration admixture of s-waves and p-waves in the ground state of ${ }^{11} \mathrm{Li}$. With a simple rotational model for the core, it is possible to obtain an effective interaction for $n-{ }^{9} \mathrm{Li}$, fitted to 
the properties of ${ }^{10} \mathrm{Li}$, that produces the two neutron binding energy, the r.m.s matter radius, the charged radius, and the structure of ${ }^{11} \mathrm{Li}$ all consistent with experiment. Core excitation in the ground state of this three-body system is found to be unimportant. This result is confirmed by shell model calculations. Finally the $\mathrm{d}$-wave strength is predicted to be very small $(\approx 7 \%)$ in the three-body model. This is in disagreement with shell model predictions that show roughly $30 \%, 50 \%, 20 \%$ for $\left(s_{1 / 2}\right)^{2},\left(p_{1 / 2}\right)^{2}$, and $d^{2}$ components. The main reason for this discrepancy comes from the corresponding states in the subsystem ${ }^{10} \mathrm{Li}$. So far, experiments have not been able to make a clear statement about d-states in ${ }^{10} \mathrm{Li}$. In our $n$ - core effective interaction we have assumed that such states would be above $3 \mathrm{MeV}$. However, shell model produces d-states that are at much lower energy (around $2 \mathrm{MeV}$ ), similar to ${ }^{11} \mathrm{Be}$. Resolving experimentally the d-states in ${ }^{10} \mathrm{Li}$ will settle once and for all the structure of the ground state of ${ }^{11} \mathrm{Li}$. This experiment should be done with a reaction starting from ${ }^{9} \mathrm{Li}$ rather than knock-out from ${ }^{11} \mathrm{Li}$ since there is not much d-wave in ${ }^{11} \mathrm{Li}$ to start with. One possibility would be to repeat the ${ }^{9} \mathrm{Li}(\mathrm{d}, \mathrm{p}){ }^{10} \mathrm{Li}$ [4] with higher beam energy.

\section{Addendum}

After the completion of this work we learned about the recent high precision mass measurement of ${ }^{11} \mathrm{Li}: S_{2 n}=376 \pm 5 \mathrm{keV}$ 42. Even though the experimental accuracy is now well beyond the accuracy of the three body model, this additional binding may be an indication that the d-wave is below $3 \mathrm{MeV}$.

\section{REFERENCES}

1. T. Kobayashi et al., Phys. Rev. Lett. 60 (1988) 2599.

2. K. Ieki et al., Phys. Rev. Letts. 70 (1993) 730.

3. M. Zinser et al., Nucl. Phys. A 619 (1997) 151.

4. B.M. Young et al., Phys. Rev. Lett. 71 (1993) 4124.

5. M.G. Gornov et al., Phys. Rev. Lett. 81 (1998) 4325.

6. P.G. Hansen, Nucl. Phys. A 553 (1993) 89c.

7. N.A. Orr et al., Phys. Rev. C 51 (1995) 3116.

8. F. Humbert et al., Phys. Letts B 347 (1995) 198.

9. M. Zinser et al., Phys. Rev. Lett. 75 (1995) 1719.

10. H. Simon et al., Phys. Rev. Lett. 83 (1999) 496.

11. A.A. Korsheninnikov et al., Phys. Rev. Lett. 78 (1997) 2317.

12. M.J.G. Borge et al., Phys. Rev. C 55 (1997) R8.

13. N. Aoi et al., Nucl. Phys. A 616 (1997) 181.

14. D.J. Morrissey et al., Nucl. Phys. A 627 (1997) 222.

15. J.S. Al-Khalili and J.A. Tostevin, Phys. Rev. Lett. 76 (1996) 3903.

16. D.R. Tilley et al., Nucl. Phys. A 745 (2004) 155.

17. G. Bertsch and H. Esbensen, Ann. Phys. 209 (1991) 327.

18. I. J. Thompson and M. V. Zhukov Phys. Rev. C 49 (1994) 1904.

19. D.V. Fedorov, E. Garrido and A.S. Jensen, Phys. Rev. C 51 (1995) 3052.

20. H. Esbensen, G.F. Bertsch and K. Kencken, Phys. Rev. C 56 (1997) 3054.

21. E. Garrido, D.V. Fedorov and A.S. Jensen, Nucl. Phys. A 700 (2002) 117. 
22. S. N. Ershov, B. V. Danilin, J. S. Vaagen, A. A. Korsheninnikov, and I. J. Thompson Phys. Rev. C 70 (2004) 054608.

23. G.F. Bertsch, B.A. Brown and H. Sagawa, Phys. Rev. C 39 (1989) 1154.

24. W. Koepf, Y.K. Gambhir, P. Ring, and M.M. Sharma, Z. Phys. A 340 (1991) 119.

25. J. Meng and P. Ring, Phys. Rev. Lett. 77 (1996) 3963.

26. S. Karataglidis, P.G. Hansen, B.A. Brown, K. Amos, and P.J. Dortmans, Phys. Rev. Letts. 79 (1997) 1447.

27. Steven C. Pieper, K. Varga, and R. B. Wiringa, Phys. Rev. C 66, 044310 (2002).

28. C. Forssén, P. Navrátil, W. E. Ormand, and E. Caurier, Phys. Rev. C 71, 044312 (2005).

29. D. Kurath, Phys. Rev. C 43 (1991) 911.

30. T.T.S. Kuo, F. Krmpotic and T. Tzeng, Phys. Rev. Lett. 78 (1997) 2708.

31. F. M. Nunes, I. J. Thompson and R. C. Johnson, Nucl. Phys. A 596 (1996) 171.

32. F. M. Nunes, J. A. Christley, I. J. Thompson, R. C. Johnson and V. D. Efros, Nucl. Phys. A 609 (1996) 43.

33. F. M. Nunes, I. J. Thompson and J. A. Tostevin, Nucl. Phys. A 703 (2002) 593.

34. F. Azenberg-Selove, Nucl. Phys. A 506 (1990) 1.

35. I.J. Thompson, F.M. Nunes, B.V. Danilin, Comp. Phys. Comm. 161 (2004) 87.

36. D. Gogny, P. Pires, R. de Tourreil, Phys. Lett. B 32 (1970) 591.

37. http://www.fresco.org.uk/programs/efaddy/

38. M. Zhukov et al., Phys. Rep. 231 (1993) 151.

39. R. Sanchez et al., Phys. Rev. Lett. 96 (2006) 033002.

40. E.K. Warburton and B.A. Brown, Phys. Rev. C 46 (1992) 923.

41. H.B. Jeppesen et al., Nucl. Phys. A 748 (2005) 374.

42. C. Bachelet et al., Eur. Phy. J. A 25 s01 (2005) 31. 